\title{
La integración de las TIC en los centros educativos: percepciones de los coordinadores y directores
}

\author{
ICT integration in educational institutions: perceptions of head teachers and coordinators
}

\section{Integração das TIC na escola na perspectiva de coordenadores e diretores}

\author{
M. Amor Pérez ${ }^{1}$, Ángel Hernando-Gómez ${ }^{2}$, \\ Ignacio Aguaded-Gómez ${ }^{3}$ \\ ${ }^{1}$ Departamento de Filología Española y sus Didácticas. \\ Universidad de Huelva. Campus del Carmen, s/n 21071 Huelva. \\ Tfno.: +34-959219114, e-mail: amor@uhu.es \\ ${ }^{2}$ Departamento de Psicología Evolutiva y de la Educación. \\ Universidad de Huelva, Facultad de Ciencias de la Educación. Campus del Carmen, s/n 21071 Huelva. \\ Tfno.: 959219429/ 610668905. Fax: 959219357, e-mail: angel.hernando@dpsi.uhu.es \\ ${ }^{3}$ Universidad de Educación y Comunicación. Departamento de Educación. \\ Universidad de Huelva. Campus del Carmen, s/n 21071 Huelva. \\ Tfno.: +34-959218295, e-mail: aguaded@uhu.es
}

\begin{abstract}
RESUMEN
En este trabajo se exponen los resultados de una investigación llevada a cabo para evaluar el impacto de la implantación de las tecnologías de la comunicación (TIC) en centros de enseñanza. La investigación ha analizado las repercusiones directas de las TIC en los procesos didácticos de enseñanza-aprendizaje utilizando para ello una variada gama de instrumentos, nos centramos en este trabajo en los resultados que proporcionan las entrevistas y los grupos de discusión, dado que éstos nos muestran, algunas de las percepciones, retos y demandas del profesorado involucrado en dicha experiencia. Se destacan como conclusiones la necesidad de consolidar la profesionalización de los equipos directivos, la relevancia del coordinador TIC como catalizador de innovaciones y la necesaria apuesta por un cambio de mentalidad del profesorado, así como de renovación de las metodologías educativas para afianzar el uso integral y didáctico de las TIC.
\end{abstract}

Palabras clave: proceso enseñanza-aprendizaje, innovación educativa, integración de las TIC, centros TIC.

\begin{abstract}
This work presents the results of an investigation that assesses the impact of information and communication technologies (ICT) on educational institutions. The research uses a wide range of instruments to analyse the direct effects of ICT on the didactic processes of teaching and learning, and the results are based on a series of interviews and group discussions that reveal the challenges, demands and perceptions of teachers involved in this experience. The main conclusions call for the need to professionalize management teams, and to stress the importance of the ICT coordinator as a conduit for new ideas. There is also a need for a change in teachers' mentality and an update in educational methodology in order to consolidate the comprehensive and didactic usage of ICT.
\end{abstract}

Key words: teaching-learning process, educational innovation, integration of ICT, ICT centers.

\section{RESUMO}

Este artigo/trabalho apresenta os resultados da pesquisa realizada nas escolas, para avaliar o impacto da implementação das Tecnologias de Comunicação e Informação (TIC). Com esta pesquisa/investigação, analisaram-se as repercussões directas das TIC, nos processos didácticos de ensino-aprendizagem, utilizando diversos instrumentos/meios para a (de) recolha de dados, com especial destaque para os resultados provenientes das entrevistas e dos grupos de discussão, dado que mostram, de forma mais objectiva e clara, algumas das 
perspectivas, desafios e exigências dos professores envolvidos nesta experiência. Como conclusões (principal conclusão), destaca-se a necessidade de consolidar (reforçar), nesta área, a profissionalização das direcções das escolas, com especial destaque do Coordenador TIC, que tem um importante papel a desempenhar como catalisador para a inovação educativa e para a mudança de mentalidades (atitudes) junto dos docentes, na renovação e/ou implementação de novas de metodologias educativas, que visem um uso global e permanente da integração das TIC no ensino.

Palavras chaves: centros TIC (temos salas TIC, centros de formação de professores), mas centros TIC concretamente não.

\section{INTRODUCCIÓN}

Este estudio es parte de una investigación realizada en Andalucía (España) para analizar la implementación del software libre en los Centros TIC andaluces y sus repercusiones en los procesos de enseñanza-aprendizaje. Dicha investigación se contextualiza en una realidad educativa que se ha ido consolidando progresivamente en todo el territorio nacional, pero también en todos los países del mundo desarrollado.

En las últimas décadas, los centros educativos de nuestro país han ido recibiendo dotaciones en hardware y software, al principio en aulas específicas, programas concretos y proyectos de carácter experimental, para ir dando paso a una inversión de carácter general desde las administraciones públicas. Este hecho se pone en evidencia en numerosos estudios de los últimos cinco años desde muy diversas perspectivas. De manera general y para todo el país, el MEC (2007), elaboró un informe extenso sobre la implantación y el uso de las TIC en los centros docentes de educación primaria y secundaria que concreta y sintetiza algunas de las características, tendencias, y resultados ante el desarrollo de los diferentes programas dirigidos a favorecer la utilización de las Tecnologías de la Información y de la Comunicación en todas las Comunidades Autónomas española (excepto País Vasco y Cataluña) y en las etapas de Educación Primaria, Educación Secundaria Obligatoria, Bachillerato y Ciclos Formativos de Formación Profesional.

A modo de somero repaso de los antecedentes de la investigación que es objeto de nuestro trabajo, la Unión Europea desarrolla desde el año 2000 iniciativas y medidas para que la utilización de las nuevas tecnologías multimedia y de Internet mejore la calidad del aprendizaje, facilitando el acceso a recursos y servicios, los intercambios y la colaboración a distancia (Sancho, 2007). En este sentido, el Grupo Operativo de Software Educativo Multimedia se crea para acelerar el desarrollo de las tecnologías de la educación y su aplicación en la Unión Europea. El Plan de acción Aprender en la sociedad de la información se implanta para fomentar la creación de redes electrónicas entre escuelas en toda Europa, estimular el desarrollo de los recursos multimedia educativos, promover la formación de los profesores sobre la utilización de las tecnologías de la información y de la comunicación e informar sobre el potencial de los instrumentos de educación audiovisual y multimedia. De esta forma, puede decirse que en Europa se genera una experiencia y unos conocimientos técnicos significativos. Sin embargo, las necesidades y expectativas de los profesores y los alumnos en las escuelas y universidades siguen suponiendo un importante desafío. Acciones como Minerva, el programa Sócrates II y el quinto Programa Marco Comunitario de Investigación y Desarrollo Tecnológico alentaban el desarrollo y la experimentación de métodos pedagógicos innovadores. 
El e-Learning ${ }^{1}$ contempla una intensificación del esfuerzo de formación mediante la promoción de una cultura digital para todos, y la generalización de programas formativos adecuados para profesores centrados en la utilización pedagógica de la tecnología y de la gestión del cambio.

En consecuencia, nuestro país y cada una de las administraciones autonómicas han desarrollado experiencias, acciones y proyectos dirigidos a la implantación e integración de las tecnologías en el ámbito educativo (Martínez Figueira, 2006; De Pablos, Area, Valverde y Correa, 2009; Sigalés, Mominó, y Meneses, 2009).

La Comunidad Autónoma de Extremadura toma la iniciativa en materia de la aplicación del software libre en la administración pública y en los centros educativos de Primaria y Secundaria en el año 2001, hecho que promueve el despegue de la política de implantación de las TIC. En el año 2003 la Comunidad Autónoma Andaluza establece las Medidas de Impulso de la Sociedad del Conocimiento en Andalucía ${ }^{2}$, que sientan las bases administrativas y jurídicas sobre las que trabajar para la universalización de las tecnologías de la comunicación y de la información. De su desarrollo se desprenden tres objetivos claros: garantizar el acceso a las TIC a todos los andaluces y andaluzas, sin discriminación de lugar de residencia, situación social o de cualquier otra cuestión; facilitar el acceso a través de Internet a la información y servicios que preste la administración (potenciando así el portal: www.andaluciajunta.es); y adaptar la prestación de servicios públicos básicos, especialmente los sanitarios y educativos, a las demandas y potencialidades de la sociedad del conocimiento.

El Proyecto de Centros TIC se inicia oficialmente el 4 de abril de 2003, cuando aparece en el Boletín de la Junta de Andalucía, regulándose la convocatoria de selección de proyectos educativos para la incorporación de las tecnologías de la información y la comunicación a la práctica docente. A partir de este año, los centros docentes andaluces comienzan a incorporar masivamente nuevos ordenadores y se integran en la Red de Centros TIC.

\section{LOS CENTROS TIC EN LA COMUNIDAD AUTÓNOMA ANDALUZA}

Los centros de tecnologías de la información y la comunicación (Centros TIC) son centros de Primaria y Secundaria que incorporan las TIC como herramienta educativa en los procesos de enseñanza-aprendizaje. La filosofía de estos centros va más allá de los usos de las tradicionales aulas de informática. Se pretende que se emplee la informática para aprender, de forma que los ordenadores se integren en las programaciones educativas como instrumentos que complementan la formación de los alumnos. De alguna

1 La iniciativa e-Learning se inscribe, por tanto, en el marco del Plan de acción global e-Europe (www.europa. eu.int/comm/information_society/eeurope/index_en.htm) que abarca el periodo 2001-2004 y pretende la movilización de los protagonistas de la educación y la formación, así como de los protagonistas sociales, industriales y económicos interesados, para hacer de la educación permanente el motor de una sociedad solidaria y armoniosa, en una economía competitiva.

2 Decreto 72/2003, de 18 de marzo, Consejería de la Presidencia de la Junta de Andalucía. La sección seis primera de los "Servicios en materia educativa", explica todo el proceso de incorporación de las tecnologías de la información y de la comunicación, los miembros implicados, las figuras relevantes en el proyecto, el material a considerar, la formación necesaria del profesorado, etc. 
manera, puede decirse que las medidas de impulso de la sociedad del conocimiento en Andalucía se concretan en el ámbito educativo. La concepción de estos centros pioneros como entornos de aprendizaje virtuales en los que se pudieran producir cambios en la forma de concebir el conocimiento, se establecieran nuevas vías de relación en y con la comunidad educativa andaluza y se formara adecuadamente al profesorado para el uso educativo de las TIC, auguraba la posibilidad de intervenir en el modelo de sociedad de la información. No obstante, nuestro estudio y otros similares (Barquín, 2005; Cebrián, Ruiz y Rodríguez, 2007; Palomo, Ruiz y Sánchez, 2007; Pérez Gómez y Sola, 2006; Colás y otros, 2006) muestran que aún queda mucho por hacer en cuanto a la consolidación de la alfabetización digital y la transformación de la educación.

En la primera convocatoria andaluza (Orden de 27-03-03 de la Consejería de Educación de la Junta de Andalucía) para seleccionar los centros que trabajarían con tecnologías de la información y de la comunicación se seleccionaron 50 centros públicos, de los que 14 pertenecían a Primaria y 36 a Secundaria Obligatoria. Estos centros fueron los pioneros en tener ordenadores integrados en redes locales conectados con banda ancha a Internet, además de ser los primeros en tener un ordenador por cada dos alumnos en las aulas, en la sala de profesores, en la administración, en la conserjería, en el equipo directivo, e incorporar el sistema operativo Guadalinex basado en Debian (Linux), elaborado por el grupo de desarrolladores informáticos de la Junta de Andalucía.

Sin embargo, lo más relevante de esta primera convocatoria de Centros TIC, salvando la dotación de infraestructura, era la necesidad de presentar "un plan general de actuaciones para la incorporación de las tecnologías a la práctica docente implicando la vida diaria del centro". En ese sentido, había que detallar: las experiencias del profesorado del centro en tecnologías de la información y la comunicación; qué persona sería la encargada de realizar las labores de coordinador o coordinadora TIC (figura fundamental en todo el proceso); motivos o circunstancias que hacían recomendable la implantación del proyecto en el centro; objetivos a corto, o largo plazo; relación de áreas de conocimiento, o materias, en las que se usarían las TIC y su nivel de utilización en cada una de ellas; y la previsión de modificaciones que deberían realizarse en el proyecto curricular del centro y en el plan anual para el correcto desarrollo del proyecto. En un apartado de la Orden se hacía mención expresa a los Centros de Profesorado para que se comprometieran en el apoyo y asesoramiento de aquellos centros que así lo solicitaran (Ávila y Tello, 2004). Todos estos aspectos indican la dimensión pedagógica y el sentido auténtico de la integración TIC, aunque los resultados de algunas de las investigaciones desarrolladas evidencian que las prácticas pedagógicas no han variado exponencialmente al amparo de la integración de las TIC (Balanskat, Blamire y Kefala, 2006; BECTA, 2007; MEC, 2007; Aguaded, 2007; Area, 2008).

\section{EXPERIENCIAS E INVESTIGACIONES SOBRE LA INTEGRACIÓN DE LAS TECNOLOGÍAS DE LA INFORMACIÓN Y LA COMUNICACIÓN}

Hasta no hace mucho los ordenadores habían entrado en los centros educativos de manera puntual o en aulas específicas. Pero ya hemos hecho notar el esfuerzo político y económico en aras de la universalización de las tecnologías de la comunicación y de la información, que se concreta en numerosas convocatorias, proyectos y programas 
amparados en la mayoría de los casos por administraciones e instituciones de rango internacional, nacional o incluso regional y local, dirigidas al ámbito educativo. Ese esfuerzo inversor en dotaciones y mantenimiento de equipos informáticos -software y hardware- a menudo no se corresponde con una adecuada política educativa que gestione de forma coherente y certera los recursos que se ponen a disposición de las escuelas. De esta forma se detectan problemas en cuanto a la adecuada implementación y aprovechamiento de los recursos, así como en lo relativo a la formación en TIC del profesorado (Hinojo y López, 2004; Castaño y otros, 2004; Tejedor y García-Valcárcel, 2006), o a la evaluación de las repercusiones de la tecnologización de las aulas en los procesos de enseñanza-aprendizaje, entre otros (Sancho, 1996, 2007; Lorenzo, Trujillo y Morales, 2007). Pese a que en las últimas décadas se han desarrollado numerosas experiencias e investigaciones en torno a la integración de las tecnologías de la información y la comunicación (TIC) en los diferentes niveles educativos, tanto en el ámbito nacional como internacional, con variadas perspectivas. En este sentido, Area (2005) señala la abundancia de "información empírica sobre las TIC en las escuelas", insistiendo en que "nos falta construir una teoría sobre este fenómeno particular de la realidad escolar que nos permita comprender qué sucede cuando los ordenadores entran en las escuelas, las causas de la resistencia del profesorado a integrar estas tecnologías en su práctica docente, o cómo implementar exitosamente estrategias de incorporación escolar de las TIC en un determinado contexto nacional o regional".

El análisis de la literatura fundamenta la evidencia científica de que la mera presencia de la informática y la telemática no es suficiente para mejorar la calidad educativa, a no ser que se apueste de forma decidida por su integración didáctica en los procesos de enseñanza-aprendizaje. En 1997, en el estudio de "Usos de los medios audiovisuales y las nuevas tecnologías en los centros andaluces", concluíamos con algunas de las ideas que nos han servido como punto de partida para este trabajo (Cabero, 2000).

La figura del docente es fundamental en el éxito de la incorporación de las TIC. Así, es definitivo lo que piensa, la actitud con que enfrenta la tecnología, su disposición a la innovación, las expectativas de éxito o fracaso hacia su integración y los posibles beneficios para el proceso de enseñanza/aprendizaje Siguiendo a Area (2005) en su revisión de las principales líneas de investigación en el ámbito de la integración de las tecnologías en el sistema escolar, los estudios sobre perspectivas, opiniones y actitudes de los agentes educativos y del profesorado hacia el uso e integración de las tecnologías tienen una importante tradición. Se pretende con ellos "disponer de un 'retrato' del estado de opinión de estos agentes educativos", o de una "diagnosis o evaluación inicial cara a planificar un programa de incorporación de nuevas tecnologías a gran escala, o cuando se pretende identificar demandas y necesidades bien formativas bien profesionales del profesorado".

En este artículo nos centramos en la perspectiva del profesorado, percepciones, retos y demandas ante esta experiencia de innovación educativa. De alguna manera nos situamos en la línea que Area (2005) ha definido como "pródiga en el contexto español" y continuamos una densa estela de trabajos que indagan en evaluaciones o análisis bien de programas concretos de integración de las TIC, o de los usos, opiniones y actitudes del profesorado en torno a cuestiones específicas o niveles educativos (Escudero, 1991; Cabero, 1991, 2000; Castaño, 1994; Castaño y Otros, 2004; Area y Correa, 1992; Colás y otros, 2006; Pérez Cota, Vilán y Machado, 2006; Pérez Gómez, y Sola, 2006; Sanabria, 
2006; Cebrián, Ruiz y Rodríguez, 2007; Aguaded y otros, 2007; Area y Otros, 2008; Area, 2008; León, Correa, Jiménez de Aberasturi, y Alex, 2008).

\section{PLANTEAMIENTO DEL PROBLEMA}

La finalidad de la investigación desarrollada ha sido analizar el impacto de las tecnologías de comunicación en los Centros TIC. A tal efecto, los objetivos que hemos contemplado en nuestro estudio han sido describir, desde una perspectiva científica, el estado actual de uso de los medios tecnológicos en dichos centros analizando la rentabilidad didáctica de su uso; obtener información contrastada sobre el grado de implementación de los nuevos recursos de software libre y su impacto en los procesos de enseñanza-aprendizaje; identificar indicadores de referencia y calidad para una integración didáctica, crítica y plural, de las tecnologías de la información en el ámbito educativo; elaborar propuestas de formación del profesorado en el ámbito de las tecnologías de la información como auxiliares didácticos, técnicas de trabajo y objetos de estudio y determinar las competencias informáticas y telemáticas claves para el desenvolvimiento ciudadano del alumnado.

\section{DISEÑO Y METODOLOGÍA DE LA INVESTIGACIÓN}

Concretado el problema y establecidas las fases del proceso, determinamos el campo, contexto físico y social en el que se realizaría el estudio. Así, seleccionamos una muestra de centros de entre los cincuenta primeros que se acogieron a la convocatoria de Proyectos TIC, en concreto estudiamos 16 centros escolares andaluces, cinco de educación Infantil y Primaria y 11 Institutos de Enseñanza Secundaria. La población sobre la que se realizó la investigación la constituyen dichos centros, en concreto 634 docentes que imparten clase en los 16 centros TIC seleccionados al azar a partir de un muestreo aleatorio por conglomerados.

Entre los procedimientos para la selección de la información, las "estrategias de recogida de datos" (Goetz y LeCompte, 1988: 124), y dependiendo de qué clase de información se pretende recopilar, cómo se va a conseguir y con qué instrumentos, optamos por emplear procedimientos muy diversos con la idea de obtener la información pertinente por distintas fuentes y vías. Así, empleamos los siguientes a la hora de recoger las informaciones: cuestionarios, entrevistas a los directores y coordinadores TIC, observaciones no participantes en las aulas, grupos de discusión y análisis de websites, plataformas y documentos. De este modo, fue posible contrastar, confirmar y triangular la información.

Este artículo, dadas las limitaciones de espacio, se centra en la aplicación de las entrevistas y los datos reportados en ellas, puesto que es el instrumento que muestra de forma más directa las percepciones de los sujetos implicados.

El material transcrito en los 16 centros objeto de esta investigación, esto es, un total de 31 entrevistas -en un centro director y coordinador eran la misma persona- confirman una amplia documentación correspondiente a los directores y coordinadores TIC que se ha codificado en 1.466 unidades y 1.278 , respectivamente: 


\begin{tabular}{|l|c|c|}
\hline \multirow{2}{*}{ Códigos } & \multicolumn{2}{|c|}{ Frecuencia } \\
\cline { 2 - 3 } & Directores & Coordinadores \\
\hline Gestación y diseño del proyecto & 276 & 216 \\
\hline Desarrollo del proyecto & 481 & 285 \\
\hline Enseñanza-aprendizaje & 163 & 189 \\
\hline Apoyo de la Administración & 189 & 261 \\
\hline Formación del profesorado & 190 & 191 \\
\hline Evaluación & 161 & 136 \\
\hline Total & 1.466 & 1.278 \\
\hline
\end{tabular}

Los testimonios que nos reportan las entrevistas muestran opiniones sinceras y más abiertas que completan los datos obtenidos con otros instrumentos en torno a qué ha supuesto la integración de las TIC en los centros. Nos centramos en aquellas categorías más significativas en relación con las percepciones, retos y demandas del profesorado.

Así, las aportaciones de directores y coordinadores, vertidas en relación con la gestación y diseño del proyecto, destacan el papel relevante que inicialmente tuvieron los equipos directivos de los centros para su puesta en marcha, así como la importancia de las iniciativas personales para la dinamización del Claustro de profesores y la comunidad educativa, que luego, por supuesto, debería ser consensuado en los diferentes órganos directivos.

Al menos por mi parte, ya que lo habrá dicho el director, el que gestó el proyecto fue él. Sinceramente fue él. Él fue el primero en que de buenas a primeras, cuando surgió lo del proyecto de Centro TIC lanzó la idea y me lo comentó a mí. Yo acepté. Él pensó que yo era la persona indicada y a partir de ahí se creó un grupo de trabajo. El director, la dirección... se empezó a trabajar y luego se propuso al Claustro (COOR08, GDI, TEXT, char 1029 to 1446 of page 1 of COORO8A.TXT).

En cuanto a la implicación de los diferentes sectores de la comunidad, en algunos casos se destaca la participación colectiva del profesorado, haciéndose referencia a la estabilidad del profesorado y al rechazo de ciertos colectivos, porque como era previsible la reacción positiva no podía ser unánime en todos los casos. Pero lo más significativo es la percepción de la figura del coordinador TIC como muy relevante.

La figura del coordinador que es quizá el líder de todo esto y luego pues al que fue director durante el curso pasado que es el actual secretario, que también muy implicado y luego a un tercer profesor que tiene una gran experiencia en la aplicación didáctica de las nuevas tecnologías (DIR16, GIR, TEXT, char 3569 to 3854 of page 1 of DIR16C.TXT).

Sus opiniones en torno a la importancia del proyecto varían en función de su visión de éste. Por un lado, están los que se han planteado el proyecto como un reto personal, por otro, aquéllos que apuntan a que los ordenadores van a ser una herramienta esencial en el futuro. La adaptación del profesorado inicialmente fue mucho más variable, generándose incluso conflictos internos que quedan bien reflejados en las manifestaciones de 
directores y coordinadores TIC. Por primera vez entraba en los centros una tecnología de la que, en muchos casos, los alumnos tenían más conocimientos que los propios docentes. Fue un auténtico reto que progresivamente fue "normalizándose" con diferentes grados de utilización.

Los primeros años había más efervescencia, había más, también más conflictividad en el sentido de que había profesores que tenían, yo creo, incluso conflictos internos de decir "yo estoy quedando como mal profesor porque yo no lo uso suficientemente esto, yo no me siento capaz, esto a mí ya me pilla tarde...", entonces yo creo que a estas alturas la cosa ha ido normalizándose (DIR09, DIP, TEXT, char 8492 to 8867 of page 1 of DIR09A.TXT).

En las entrevistas se recogen incidencias negativas y problemas derivados de la implementación del Proyecto: temporalización, mal funcionamiento de los equipos, configuraciones, mobiliarios..., además de expectativas positivas en la comunidad educativa.

Todo empezó a funcionar mal, no iba Internet, los equipos no funcionaban, problemas con las tarjetas gráficas que hacían que la señal no pasase al monitor... pues eso ocurría creo que de 500 equipos pues en 300. No existía asistencia técnica en aquel entonces. Entonces, equipo que se estropeaba, equipo que no sabíamos qué hacer con él (COOR15, DIP0, TEXT, char 5657 to 5991 of page 1 of COOR15A.TXT).

Un sector mayoritario afirma que el Proyecto TIC ha generado muchas transformaciones deseadas, a nivel de organización y especialmente en el ámbito curricular. Así, exponen que la llegada de los aparatos supuso la aparición de variables que antes no se habían contemplado, afectando a múltiples aspectos como el papel del profesor en el aula, la organización de la clase, las entradas y salidas para el control de los equipos, los reglamentos de funcionamiento, etc. En algunos centros se han creado incluso los "delegados TIC".

Otro cambio, por ejemplo, ha obligado a crear una serie de documentos para el control del funcionamiento de las aulas, de las incidencias del mal funcionamiento que se produzcan en los ordenadores, que haya mecanismos para control (DIR09, DCC, TEXT, char 8721 to 9017 of page 1 of DIR09B.TXT).

Luego tenemos la figura de los delegados TIC, que son alumnos, para que informen al tutor $y$ a mí de las incidencias (COOR14, DCC1, TEXT, char 4197 to 4312 of page 1 of COOR14B. TXT).

También comentan cómo los ordenadores han exigido cambios en las dinámicas de alumnos y profesores, con nuevas maneras de enseñar y aprender antes insospechadas, o las necesidades formativas del profesorado, que se reivindican muy frecuentemente.

Las necesidades de formación del profesorado es otro de los factores esenciales en el cambio. Sí hay un incremento en ese sentido y además también un incremento; vamos un incremento no, un pasar de cero a que haya algo, en lo que se refiere a organización de la formación del profesorado (DIR09, DCC1, TEXT, char 6073 to 6266 of page 1 of DIR09B.TXT).

Se reitera que la figura del coordinador TIC es central en la experiencia y se confirma el menoscabo de este papel esencial en la dinamización docente, convertido en mero apoyo técnico por las circunstancias. Así, en numerosas ocasiones los directores 
y coordinadores demandan el carácter pedagógico o formativo y argumentan que esta aspiración sólo se va plasmando en la medida en que se van resolviendo los problemas técnicos, ofreciéndose la asesoría didáctica en algunos casos como impartición de cursos a los colegas y en otros casos como atención individualizada. Para muchos coordinadores, la presencia del técnico informático en los centros es indiscutible.

El perfil que dibuja el proyecto es el de la persona que dinamiza el uso de los recursos. Y la realidad es que hay tal cantidad de incidencias de tipo técnico que son primarias porque si los ordenadores no funcionan todos en un aula... ¿cómo te vas a poner a dinamizar?; que hacen que el coordinador TIC al final de cuentas es el responsable del mantenimiento de los equipos (DIR09, DCO, TEXT, char 5455 to 5827 of page 1 of DIR09C.TXT).

Yo intento que mi labor sea pedagógica, exclusivamente pedagógica: apoyar a los compañeros a la hora de enfocar líneas de trabajo (COOR02, DCO, TEXT, char 1780 to 1909 of page 1 of COORO2D.TXT).

En muchos casos, refieren los enormes beneficios que el proyecto de Centro TIC ha generado en la vida de los colegios e institutos: sobredotación de infraestructura informática, mobiliario... Ellos lo califican abiertamente como una revolución, especialmente para aquellos centros de partida peor dotados, manifestando incluso que la asignación económica para funcionamiento creció exponencialmente. También se incide en que plantear de partida que todas las aulas del centro tengan una configuración TIC es un error ya que en muchas materias y en ciertos contextos no es necesario este uso y la presencia de los equipos impide el desarrollo de la clase, fundamentalmente por la rigidez del mobiliario y las mesas ocupadas por un monitor CRT.

Yo creo que el proyecto tiene un problema de base, yo creo que no es recomendable el que haya, como en este centro, 32 aulas con ordenadores, cuando no hay setenta y tantos profesores que están sacándole partido a eso. Yo creo que con que hubiesen habido ocho aulas, y con unos cuadrantes para utilizarlas, se utilizarían mucho más, mucho mejor los recursos y yo creo que sería bastante mejor. Y sobre todo, haber puesto otro modelo de equipos (COORO7, DINO, TEXT, char 2756 to 3198 of page 1 of COOR07C.TXT).

Lo que más defecto le veo a las TIC es la rigidez en la estructura de la clase. Yo, por ejemplo, soy muy partidario de crear clases en " $L$ ", o hacer grupos de trabajo, y no se puede porque las mesas están ancladas al suelo. Entonces, eso sí lo ha echado mucho de menos el profesorado a la hora de trabajar en grupos (COOR04, DESO, TEXT, char 4447 to 4762 of page 1 of COORO4B.TXT).

Los coordinadores y directores de los centros TIC realizan interesantes manifestaciones en relación con lo que hemos categorizado como "enseñanza-aprendizaje", considerando el uso concreto en materias y clases, referencias a los cambios en las formas de enseñar y aprender, y valoraciones acerca del grado de aprendizaje del alumnado. Directores y coordinadores, destacan la motivación que las TIC generan en los alumnos, el acceso rápido a la información, las nuevas herramientas interactivas (webquest, buscadores, diccionarios, enciclopedias, etc.), y su uso en prácticamente todas las materias. Sin embargo, no todos tienen el mismo nivel de implicación y, por tanto, el uso es mucho más limitado, y algunos hacen hincapié en el tema del control de la clase por parte del profesor, reclamándose incluso algún tipo de dispositivo para controlar los equipos de los alumnos. 
El hecho de que un profesor cuando se pone a planificar su docencia se plantee a ver en qué elementos puedo yo utilizar bien una actividad de Internet, bien una presentación, bien una actividad con cualquier otra herramienta, tal, ya eso implica un cambio. Eso sí ha ocurrido en buena parte del profesorado (DIR15, EUT, TEXT, char 5660 to 5966 of page 1 of DIR15D.TXT).

No es que haya que coger los ordenadores todo el día y a todas horas y a cada segundo, no es eso. Yo siempre digo lo mismo: esto es un recurso más que tenemos, y para mí el recurso más importante ¿por qué? Porque el propio ordenador me lo está dando todo (COOR12, EUT, TEXT, char 4532 to 4787 of page 1 of COOR12B.TXT).

En general, coinciden en que su uso didáctico crece de forma progresiva, y matizan cómo los ordenadores han modificado la interacción en el aula con los libros, convirtiéndose en algunos casos en fuentes alternativas de conocimiento, complementados con el uso de otras tecnologías como el videoproyector, ordenador y la comunicación a través de la plataforma y el correo.

Cada vez vas utilizando más el ordenador, cada vez vas intercalando más herramientas nuevas en tu práctica y cada vez vas necesitando menos lo que antes era la práctica cotidiana, cada vez vas utilizando menos la pizarra, cada vez vas dependiendo menos de las actividades de un libro de texto (DIR02, ECA, TEXT, char 1119 to 1411 of page 1 of DIRO2B.TXT).

Hemos ido modificando nuestro concepto, cada uno en su medida, de la docencia (DIR15, ECA, TEXT, char 510 to 586 of page 1 of DIR15C.TXT).

Coordinadores TIC y directores afirman que hay que ser cautos a la hora de valorar los cambios que se han producido en los procesos de enseñanza aprendizaje. Muchos se manifiestan optimistas ante el amplio y variado conjunto de transformaciones que ha generado la implantación del Proyecto, no sólo por el aumento del uso de la informática, sino por la incorporación de nuevas herramientas para que los alumnos trabajen de forma más independiente. Incluso algunos van más allá y señalan que se empieza a producir un cambio de mentalidad, un cambio estructural en la metodología de enseñar y de aprender, en la que la adquisición de conocimiento tiene otras fuentes de acceso. Indican que lo importante no son los ordenadores para que haya un cambio sustancial, sino los profesores que sean capaces de establecer una metodología activa y acorde con las tecnologías.

El avance didáctico o educativo del uso de las herramientas sinceramente yo creo que todavía está por ver (DIR16, ECA, TEXT, char 4401 to 4507 of page 1 of DIR16A.TXT).

El cambio metodológico que hubiese sido ir prescindiendo del libro del texto y pasarse a las nuevas tecnologías, pero claro, ninguno estaríamos dispuestos a decidirlo de hoy para mañana. Te deberían de formar, entonces lo que se ha hecho es compaginar lo tradicional con una nueva herramienta (DIR13, ECA, TEXT, char 11430 to 11722 of page 1 of DIR13D.TXT).

Entre otras manifestaciones, se reitera el debate sobre el papel del libro en la educación, la necesidad de tiempo y esfuerzo suplementario para la elaboración de materiales $\mathrm{y}$, especialmente, una intensa y sólida formación del profesorado que ayude a superar un cierto miedo al vacío y a lo desconocido, presente en muchos docentes.

Vienen muchos compañeros míos diciéndome que tenemos que cambiar cosas, que los libros de texto tienen un papel secundario en algunas áreas (COOR03, ECA, TEXT, char 3815 to 3955 of page 1 of COORO3B.TXT). 
Creo que el cambio de mentalidad (DIR14, ECA, TEXT, char 8724 to 8755 of page 1 of DIR14C.TXT).

Muchos perciben los ordenadores como una inyección de moral, una ventana abierta que genera otras habilidades hasta ahora adormecidas: reflexividad, colaboración, sistemas de búsqueda... esto es, un conjunto de instrumentos que les van a permitir "vivir mejor" y acorde con los nuevos tiempos. El saber se ve ahora más redistribuido, los alumnos interactúan con el profesor, se generan nuevos ritmos de clase, hay una mayor autonomía en la búsqueda y tratamiento de la información... parecen que las tecnologías por primera vez han abierto la puerta de par en par hacia una enseñanza más activa y colaborativa.

No es tan importante el número de conocimientos que adquieran, sino los instrumentos que se les pueden facilitar para vivir mejor. Las TIC son una herramienta mejor para adaptarse a la nueva sociedad, como el ferrocarril lo fue en su tiempo (DIR05, ECA1, TEXT, char 2370 to 2610 of page 1 of DIR05B.TXT).

Se ve que el maestro no es el único conocedor del saber. El alumno construye su propio conocimiento y eso es muy importante (DIR05, ECA1, TEXT, char 5177 to 5300 of page 1 of DIR05B.TXT).

En general, consideran que el Proyecto ha generado mejoras en los alumnos aunque con condiciones. Hay un cierto escepticismo por parte del profesorado respecto a las posibilidades que aportan las tecnologías en el proceso de enseñanza-aprendizaje, de manera que las opiniones se mueven entre las dudas, la prudencia a la hora de valorar su papel o el desconocimiento de lo que éstas pueden deparar. En todo caso, denotan preocupación e interés al mismo tiempo.

Desde el punto de vista didáctico todavía no tenemos datos totalmente fehacientes de que esto esté mejorando digamos, de modo apreciable, los resultados académicos, porque es que tiene ventajas pero también tiene sus inconvenientes (DIR07, EGR, TEXT, char 10243 to 10474 of page 1 of DIRO7A.TXT).

Yo creo que se está facilitando el camino para que los niños aprendan más (DIR01, EGR1, TEXT, char 4950 to 5023 of page 1 of DIR01B.TXT).

\section{CONCLUSIONES}

Los resultados del análisis de las percepciones, retos y demandas del profesorado de los centros TIC nos llevan a concluir que:

- Los proyectos para una integración didáctica, crítica y plural de las tecnologías de la información en el ámbito educativo surgen a iniciativa del equipo directivo, así como, al menos, de un grupo de profesores que esté convencido de la iniciativa, contando con el apoyo de la Administración y del claustro. Normalmente estos proyectos se desarrollan gracias al empuje de un pequeño grupo de colegas que con su esfuerzo $\mathrm{y}$, generalmente, liderados por el coordinador TIC, difunden y propagan acciones y experiencias que acaban generalizándose.

- Los planes no pueden limitarse a la dotación de materiales, equipamiento, mobiliario y apoyo técnico. Esta medida técnica en su apariencia, pero pedagógica en el fondo, es 
un aspecto que puede mejorarse a través de mecanismos que faciliten la implicación del profesorado y mantengan su entusiasmo motivados ante los resultados.

- La figura del coordinador TIC como dinamizador de procesos de innovación didáctica, resulta esencial e imprescindible para el impulso de estos proyectos. Sin embargo, la falta de apoyos, recursos y medidas de acompañamiento ha hecho que su función se pervierta y su labor se incline fundamentalmente a tareas de asistencia técnica en perjuicio de la dinamización de grupos de trabajo. Tal situación acaba desilusionando y provocando, en muchas ocasiones, el abandono del puesto.

- El uso de las TIC ha supuesto, hasta el final de nuestro trabajo pocos cambios metodológicos. Los procesos de enseñanza-aprendizaje se han modificado porque las clases se vuelven más activas y participativas facilitando el aprendizaje autónomo de los alumnos, generándose otras habilidades: reflexión, colaboración, sistemas de búsqueda, mayor autonomía en la búsqueda y tratamiento de la información... Se ha producido un tránsito hacia una cultura cooperativa, caracterizada por una mayor colaboración y comunicación entre los profesores, un aumento del sentimiento de unidad ante un reto común, asumiéndose una responsabilidad compartida ante el reto organizativo que supone rentabilizar el equipamiento y materiales tecnológicos del centro y alcanzar los objetivos formulados y replanteados en los Proyectos TIC.

- El profesorado percibe las TIC como recursos valiosos por su potencial didáctico y educativo, aunque afirma que el uso suele ser irregular, puntual y discontinuo. No obstante, la utilización de medios tecnológicos provoca un aumento de la motivación del alumnado que se traduce en una actitud más favorable hacia las tareas académicas propuestas por el profesorado, mejorando la atención a sus indicaciones y su implicación en el aprendizaje.

- No podemos considerar los efectos del uso de las TIC en los centros docentes hasta que no se produzca una verdadera integración normalizada de las mismas en las dinámicas de aula, circunstancia que aún no es observable de forma sistemática. En todo caso, el aprovechamiento didáctico de estos recursos y las consecuencias favorables que puedan tener conlleva un giro en la mentalidad del profesorado, circunstancia que, como algunos directores de centro afirman, está comenzando a producirse. Se trata de un cambio en la manera de entender la enseñanza, desde la que el conocimiento tiene múltiples fuentes de acceso. El conocimiento comienza a verse más distribuido, los alumnos interactúan con el profesor y se generan nuevos ritmos de clase.

- El cambio de mentalidad del profesorado no está resultando gratuito, dado que un proyecto de esta envergadura, aparte de las dotaciones y servicios ofrecidos por la Administración educativa, exige tiempo al profesorado para implicarse en los procesos formativos del centro, la elaboración y búsqueda de materiales adaptados, tareas a menudo nada fáciles.

Finalmente, consideramos que resulta necesario establecer programas, recursos humanos y logísticos que faciliten el proceso de normalización del uso de las TIC en los centros docentes, no ya como un recurso más sino como vehículo hacia un cambio de paradigma didáctico y educativo, en el que las opiniones y experiencias del profesorado tienen mucho que aportar. 


\section{REFERENCIAS BIBLIOGRÁFICAS}

Aguaded J.I. y Otros (2007). "Observatics”: La implementación del software libre en Centros TIC andaluces. Análisis de las repercusiones en los procesos de enseñanza-aprendizaje. Huelva: Universidad de Huelva.

Anderson, R.E. (2002). Guest editorial: international studies of innovative uses of ICT in schools. Journal of Computer Assisted Learning, $\mathrm{n}^{\circ} 18$ (4); 381-386.

Area, M. (2008). Innovación pedagógica con TIC y el desarrollo de las competencias informacionales y digitales. Investigación en la escuela, $\mathrm{n}^{\circ} 64 ; 5-18$.

Area, M. y Correa, A.D. (1992). La investigación sobre el conocimiento y actitudes del profesorado hacia los medios. Una aproximación al uso de medios en la planificación y desarrollo de la enseñanza, en Qurrículum, no 4, 79-100.

Ávila, J.A. y Tello, J. (2004). Reflexiones sobre la integración curricular de las tecnologías de la comunicación, en Comunicar, $\mathrm{n}^{\circ}$ 22, 177-182.

Barquín, J. (2005). La implantación de las Tecnologías de la Información en la sociedad y en los centros educativos públicos de la Comunidad de Andalucía. Revista Iberoamericana de educación, no $36,155-174$.

Cabero, J. (1991). Actitudes de los profesores hacia los ordenadores y la informática. En Cebrián, M. (Dir.). Medios y recursos didácticos. Málaga: Secretariado de Publicaciones de la Universidad de Málaga.

Cabero, J. (Dir.) (2000). Uso de los medios Audiovisuales, informáticos y las NNTT en los centros andaluces. Sevilla: Kronos.

Castaño, C. (1994). Análisis y evaluación de las actitudes de los profesores hacia los medios de enseñanza. Bilbao: Universidad del País Vasco.

Castaño, C. y Otros (2004). La utilización de las TIC en la enseñanza primaria y secundaria obligatoria: necesidades de formación del profesorado. Congreso EDUTEC 2004, De lo excepcional a lo cotidiano. Barcelona: EDUTEC.

Cebrián, M., Ruiz, J. y Rodríguez, J. (2007). Estudio del impacto del Proyecto TIC desde la opinión de los docentes y estudiantes en los primeros años de su implantación en los centros públicos de Andalucía. Málaga: Universidad de Málaga, Grupo de Investigación en Nuevas Tecnologías aplicadas a la Educación.

Cohen, L.M., Manion, L. \& Morrison, K. (2003). Research Methods in Education. London: Routledge Falmer.

Colas, P. y Otros (2006). Incorporación de las TIC en los Centros de Secundaria y Bachillerato. Sevilla: Consejería de Educación, Ciencia y Tecnología.

De Pablos, J., Area, M., Valverde, J. y Correa, J.M. (2009). Autonomic educational policies and the integration of ICT in school centers. En A. Méndez-Vilas, A. Solano Martín, J.A. Mesa González and J. Mesa González Research, Reflections and Innovations in Integrating ICT in Education. Badajoz: Formatex.

Escudero, J.M. (1991). Evaluación de los Proyectos Atenea y Mercurio. Encuentros Nacionales sobre las Nuevas Tecnologías en la Educación (245-267). Santander: ICE de la Universidad de Cantabria.

Fuentes, J.A., Ortega. J.A. y Lorenzo, M. (2005). Tecnofobia como déficit formativo. Investigando la integración curricular de las TIC en centros públicos de ámbito rural y urbano. Educar, $36 ; 169-180$.

Goetz, J. y LeCompte, M. (1988). Etnografía y diseño cualitativo en investigación educativa. Madrid: Morata.

Hinojo, F.J. y López, J.A. (2004). Instrumentos de diagnóstico para la formación docente en tecnologías, en Comunicar, $\mathrm{n}^{\circ}$ 23, 160-165. 
León, M., Correa, J.M., Jiménez de Aberasturi, E. y Alex Ibáñez, A. (2008). La gestión del cambio tecnológico en los centros educativos del País Vasco: El papel de los directivos escolares. Pixel-Bit: Revista de Medios y Educación, $\mathrm{n}^{\circ}$ 31; 39-57.

Lorenzo, M., Trujillo, J.M. y Morales, O. (2008). Los equipos directivos de educación primaria ante la Integración de las TICs. Pixel-Bit. Revista de Medios y Educación, 33; 91-110.

Palomo, R., Ruiz, J. y Sánchez, J. (2007). Las TIC como agente de innovación educativa. Sevilla, Junta de Andalucía. Consejería de Educación, Dirección General de Innovación Educativa y Formación del Profesorado.

Pérez Cota, M., Vilán L. y Machado da Costa, J.P. (2006). Integración de las TIC en el sistema educativo de Galicia: respuesta de los docentes. RELATEC: Revista Latinoamericana de Tecnología Educativa, Vol. 5, n 2, 177-189.

Pérez Gómez, A.I. y Sola, M. (2006). La emergencia de buenas prácticas. Informe final. Junta de Andalucía, Consejería de Educación. Dirección General de Innovación Educativa y Formación del Profesorado. Sevilla.

Sancho, J.M. (2007). El (difícil) papel de las TIC en la personalización de la enseñanza, en Organización y Gestión Educativa: Revista del Fórum Europeo de Administradores de la Educación, vol. 15, n 4, 30-34.

Sancho, J.M. (1996). Aprendizaje y tecnologías de la información y la comunicación, en Kikiriki, $n^{\circ} 42-43,90-95$.

Taylor, S. y Bogdan, R. (1996). Introducción a los métodos cualitativos de investigación: la búsqueda de significados. Barcelona: Paidós.

Tejedor, F. y García-Valcárcel, A. (2006). Competencias de los profesores para el uso de las TIC en la enseñanza: análisis de sus conocimientos y actitudes. Revista española de pedagogía, 64,$233 ; 21-43$.

\section{FUENTES ELECTRÓNICAS}

Area, M. (2005). Las tecnologías de la información y comunicación en el sistema escolar. Una revisión de las líneas de investigación. Revista Electrónica de Investigación y Evaluación Educativa, v. 11, n. 1. Recuperado el 11 de enero de 2011, de: www.uv.es/RELIEVE/v11n1/ RELIEVEv11n1_1.htm.

Area, M. y Otros (2008). La integración y uso de las Tecnologías de la Información y Comunicación en los centros educativos. Análisis de casos en Canarias. Resumen ejecutivo. Recuperado el 13 de febrero de 2011, de: http://webpages.ull.es/users/manarea/RESUMENProyectoTICCanarias. pdf.

Balanskat, A., Blamire. R. y Kefala, S. (2006). The ICT Impact Report: A Review of Studies of ICT Impact on Schools in Europe. Recuperado el 10 de agosto de 2008, de: http://insight.eun. org/ww/en/pub/insight/misc/specialreports/impact_study.htm

Becta (2006). The Becta Review 2006: Evidence On The Progress Of ICT In Education. UK: Becta. Recuperado el 10 de febrero de 2011, de: http://becta.org.uk/corporate/publications/ documents/The_Becta_Review_2006.pdf.

Fernández, S. y Lázaro, M.N. (2008). Coordinador/a TIC. Pieza clave para la integración de las nuevas tecnologías en las aulas. Revista Latinoamericana de Tecnología Educativa, no 7 (2), 177-187. Recuperado el 14 de enero de 2011, de: http://campusvirtual.unex.es/cala/editio/.

Martínez Figueira, $M^{a}$.E. (2006). Políticas autonómicas para la integración de las TIC en centros educativos, Revista Latinoamericana de Tecnología Educativa, 5 (2), 97-112. Recuperado el 14 de febrero de 2011, de: www.unex.es/didactica/RELATEC/sumario_5_2.htm.

MEC (2007). Las tecnologías de la información y de la comunicación en la educación. Informe sobre la implantación de las TIC en los centros docentes de Educación Primaria y Secundaria 
(2005-2006). Ministerio de Educación y Ciencia. Red.es. Plan Avanza. Recuperado el 14 de enero de 2011, de: www.ontsi.red.es/educacion/articles/id/2605/informe-sobre-implantacionuso-las-tic-los-centros-docentes-educacion-primaria-secundaria-curso-2005-2006.html.

Sanabria, A.L. (2006). Las TIC en el sistema escolar de Canarias: los programas institucionales de innovación educativa para la integración curricular de las Tecnologías de la Información y la Comunicación, Revista Latinoamericana de Tecnología Educativa, 5 (2), 191-202. Recuperado el 14 de febrero de 2011, de: www.unex.es/didactica/RELATEC/ sumario_5_2.htm.

Sigalés C., Mominó, J.M. y Meneses, J. (2009). TIC e innovación en la educación escolar española: estado y perspectivas. TELOS. Cuadernos de Comunicación e Innovación, 78; Dossier La escuela digital. Desafíos de la innovación educativa. Recuperado el 10 de enero de 2011, de: http://sociedadinformacion.fundacion.telefonica.com/telos/articulocuaderno.asp@ idarticulo $=4 \&$ rev $=78 . \mathrm{htm}$.

Sigalés, C., Mominó, J.M., Meneses, J. y Badia A. (2008). La integración de Internet en la educación escolar española: situación actual y perspectivas de futuro. Informe de Investigación. Universitat Oberta de Catalunya, Fundación Telefónica. Recuperado el 9 de enero de 2011, de: www.fundacion.telefonica.com/. 
